\title{
IRRATIONALITY MEASURES FOR CONTINUED FRACTIONS WITH ASYMPTOTIC CONDITIONS
}

\author{
Jaroslav HANČL and Kalle LEPPÄLÄ \\ (Received 10 April 2015 and revised 2 October 2015)
}

\begin{abstract}
We are interested in lower and upper bounds of asymptotic irrationality measures for certain simple continued fractions. A general procedure for estimation of real numbers by rational numbers is described. It is applied for simple continued fractions with quotients satisfying some asymptotic density conditions.
\end{abstract}

\section{Introduction}

Standard definitions we use include the following.

Throughout the entire paper we denote by $\mathbb{Z}, \mathbb{Z}^{+}, \mathbb{N}, \mathbb{R}$ and $\mathbb{R}^{+}$the set of all integers, positive integers, non-negative integers, real numbers and positive real numbers, respectively. Square brackets $a=\left[a_{0} ; a_{1}, \ldots\right]$ stand for the simple continued fraction expansion of the real number $a$, and the numerator and the denominator of the $n$th convergent $\left[a_{0} ; a_{1}, \ldots, a_{n}\right]$ are denoted by $p_{n}$ and $q_{n}$, respectively. The notation $f(x)=O(g(x))$ and $f(x)=o(g(x))$ mean that

$$
\limsup _{x \rightarrow \infty,(f(x), g(x)) \neq(0,0)}\left|\frac{f(x)}{g(x)}\right| \neq \infty
$$

and

$$
\lim _{x \rightarrow \infty} \frac{f(x)}{g(x)}=0,
$$

respectively. By $\log x$ we mean the natural logarithm of the number $x$ and we use the convention $0^{0}=1$. If $x$ is a real number then $[x]$ is the integer part of the number $x$. The number of positive divisors of $n$ is denoted by $d(n)$.

Following Euler [4], Perron [16], Mahler [14] and Bundschuh [3] we prove the following.

THEOREM 1.1. Let $a=\left[a_{0} ; a_{1}, \ldots\right]$ be a continued fraction and let $\varepsilon$ be a positive real number. Let $\left\{V_{n}\right\}_{n=0}^{\infty}$ be a sequence consisting of numbers 1 and 2 such that $\lim _{n \rightarrow \infty}(1 / n)\left(\#\left\{j: V_{j}=1,1 \leq j \leq n\right\}\right)=\frac{1}{2}$. Set $a_{n}=n^{V_{n}}$ for all $n \in \mathbb{N}$. Then for every $M \in \mathbb{Z}$ and sufficiently large $N \in \mathbb{Z}^{+}$we have

$$
\left(\frac{9}{4}-\varepsilon\right)\left(\frac{\log \log N}{N \log N}\right)^{2}<\left|a-\frac{M}{N}\right|
$$

and for infinitely many $(M, N) \in \mathbb{Z} \times \mathbb{Z}^{+}$we have

$$
\left(\frac{9}{4}+\varepsilon\right)\left(\frac{\log \log N}{N \log N}\right)^{2}>\left|a-\frac{M}{N}\right|
$$

2010 Mathematics Subject Classification: Primary 11K60, 11J82, $11 \mathrm{~J} 70$.

Keywords: continued fraction; Diophantine approximation; irrationality measure.

(C) 2016 Faculty of Mathematics, Kyushu University 
This kind of problem has a long history which starts from Euler [4]. In 1998 Bundschuh [3] proved that

$$
\left|a-\frac{M}{N}\right| \geq \frac{D \log \log N}{d N^{2} \log N}\left(1-\frac{\log \log n}{\log n}+O\left(\frac{1}{\log n}\right)\right),
$$

where $N=q_{n}$ is the denominator of the $n$th convergent of the continued fraction

$$
a=\left[a_{0} ; a_{1}, \ldots, a_{s}, \overline{c_{1}+\lambda d_{1}, \ldots, c_{m}+\lambda d_{m}}\right]_{\lambda=0}^{\infty}
$$

satisfying the conditions

$$
a_{0}, a_{1}, \ldots, a_{s}, c_{1}, \ldots, c_{m} \in \mathbb{Z}^{+}, \quad d_{1}, \ldots, d_{m} \in \mathbb{N}, \quad d=\max \left(d_{1}, \ldots, d_{m}\right) \neq 0,
$$

and $D$ is the number of the non-zero $d_{j}$. Later Tasoev [19] proved that if $\varepsilon$ is a positive real number then for every $M \in \mathbb{Z}$ and sufficiently large $N \in \mathbb{Z}^{+}$we have

$$
\left(\frac{D}{d}-\varepsilon\right)\left(\frac{\log \log N}{N^{2} \log N}\right)<\left|a-\frac{M}{N}\right|
$$

and for infinitely many $(M, N) \in \mathbb{Z} \times \mathbb{Z}^{+}$we have

$$
\left(\frac{D}{d}+\varepsilon\right)\left(\frac{\log \log N}{N^{2} \log N}\right)>\left|a-\frac{M}{N}\right| .
$$

In the same paper, Tasoev also proved that if $a_{0}, b$ and $m$ are positive integers such that $b>1$,

$$
a=[a_{0} ; \underbrace{\overline{b^{\lambda}, \ldots, b^{\lambda}}}_{m \text { times }}]_{\lambda=1}^{\infty}
$$

and $\varepsilon$ is a positive real number, then for every $M \in \mathbb{Z}$ and sufficiently large $N \in \mathbb{Z}^{+}$we have

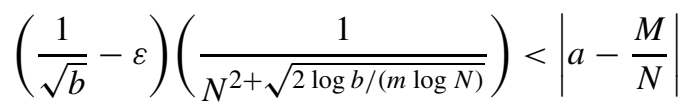

and for infinitely many $(M, N) \in \mathbb{Z} \times \mathbb{Z}^{+}$we have

$$
\left(\frac{1}{\sqrt{b}}+\varepsilon\right)\left(\frac{1}{N^{2+\sqrt{2 \log b /(m \log N)}}}\right)>\left|a-\frac{M}{N}\right| .
$$

We improve this result in Theorem 3.7 by proving that there exists a positive real number $H$ such that for every $M \in \mathbb{Z}$ and sufficiently large $N \in \mathbb{Z}^{+}$we have

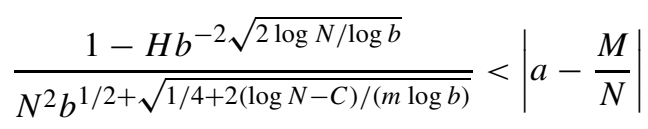

and for infinitely many $(M, N) \in \mathbb{Z} \times \mathbb{Z}^{+}$we have

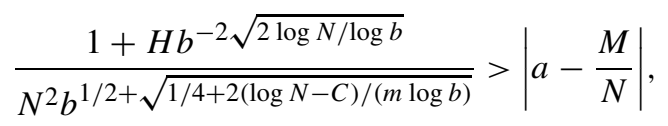

where

$$
e^{C}=b \prod_{j=2}^{\infty}\left(1+\frac{q_{j-2}}{a_{j} q_{j-1}}\right)
$$

is a constant and $q_{n}$ is the denominator of the $n$th convergent of $a$. 
Number $e$ has a simple continued fraction expression like $a$ has in (1). Many mathematicians used this fact to prove irrationality measures of $e$. We can find this in the papers of Bundschuh [2], Galochkin [6] and Komatsu [13]. See also Borwein and Borwein [1], Fel'dman and Nesterenko [5], and Shiokawa [17]. Better approximation of the number $e$ we can find in Hančl et al [7] or in [9]. Also Sondow [18] obtained a new kind of irrationality measure for $e$. Hata [12] proved an interesting approximation of $e^{n}$. Mahler [14] approximated exponential and logarithmic functions.

Compared to the number $a$ in (1), which has an almost periodic continued fraction expression, we work with asymptotical conditions only. This is connected to an earlier work of Hančl et al [10] using the concept of asymptotic density determining the Hausdorff dimension of special sets of continued fractions from the interval $(0,1)$. Theorems 3.1-3.3 deal with continued fractions with quotients $a_{n}$ growing somewhat linearly, as in (1). In Theorem 3.1, we only assume that $a_{n} / n$ has finitely many accumulation points, and that they are positive and finite. The results of Theorems 3.2 and 3.3 are more exact but here we assume that we know the asymptotic densities or even the accumulation points. In the more general Theorems 3.4 and 3.5, we make some assumptions on the average behaviour of $\log a_{n} / \log n$. This extends the results in the modern mathematical theory of means when the function is irregular but the average of this function exists and can be simply expressed. One example of this is Theorem 3.6 where we study a continued fraction constructed using the divisor function $d(n)$, the average and maximal behaviour of which is known. More examples, including a refined version of Theorem 3.6, can be found in [8].

\section{Notation}

Some less-known notation we use is as follows.

For a real number $a$ we define the function $J_{a}: \mathbb{Z}^{+} \rightarrow \mathbb{R}_{0}^{+}$as

$$
J_{a}(N)=N^{2} \min _{M \in \mathbb{Z}}\left|a-\frac{M}{N}\right| .
$$

This is related to the concepts of the irrationality measure of $a$, which usually means any lower bound of $J_{a}(N) / N^{2}$, and the irrationality exponent of $a$, which is

$$
\mu_{a}(N)=2-\liminf _{N \rightarrow \infty} \frac{\log J_{a}(N)}{\log N} .
$$

For convenience of notation we say that $J_{a}(N)=f(N)+\bar{O}(g(N))$ if there exists $C \in \mathbb{R}^{+}$ such that:

(1) $\quad J_{a}(N)>f(N)-C|g(N)|$ for all $N \in \mathbb{Z}^{+}$big enough;

(2) $J_{a}(N)<f(N)+C|g(N)|$ for infinitely many $N \in \mathbb{Z}^{+}$.

Similarly we define that $J_{a}(N)=f(N)+\bar{o}(g(N))$ if for every $\varepsilon \in \mathbb{R}^{+}$:

(1) $J_{a}(N)>f(N)-\varepsilon|g(N)|$ for all $N \in \mathbb{Z}^{+}$big enough;

(2) $J_{a}(N)<f(N)+\varepsilon|g(N)|$ holds for infinitely many $N \in \mathbb{Z}^{+}$.

In other words, we do not only provide irrationality measures but also show how sharp they are. Let $a_{0}$ be an integer, $a_{1}, \ldots, a_{s}$ be positive integers and $F_{j}: \mathbb{Z}^{+} \rightarrow \mathbb{Z}^{+}, j=1, \ldots, m$ 
be functions. Then we denote

$$
\begin{aligned}
& {\left[a_{0} ; a_{1}, \ldots, a_{s}, \overline{F_{1}(\lambda), \ldots, F_{m}(\lambda)}\right]_{\lambda=1}^{\infty}} \\
& \quad=\left[a_{0} ; a_{1}, \ldots, a_{s}, F_{1}(1), \ldots, F_{m}(1), F_{1}(2), \ldots, F_{m}(2), \ldots\right] .
\end{aligned}
$$

The reader will also need to know some basic facts about simple continued fractions. Let $a=\left[a_{0} ; a_{1}, \ldots\right]$. For the convergents we have the recurrence formulae

$$
p_{n+2}=a_{n+2} p_{n+1}+p_{n}, \quad q_{n+2}=a_{n+2} q_{n+1}+q_{n}
$$

for all $n \in \mathbb{N}$, with the initial values $p_{0}=a_{0}, q_{0}=1, p_{1}=a_{1} a_{0}+1$ and $q_{1}=a_{1}$. It is a generally known fact that the best approximations of the number $a$ are its convergents. In fact, $p / q$ is a convergent of $a$ whenever $|a-p / q|<1 /\left(2 q^{2}\right)$, and for the convergents we have

$$
\frac{1}{q_{n}^{2}\left(a_{n+1}+2\right)}<\left|a-\frac{p_{n}}{q_{n}}\right|<\frac{1}{q_{n}^{2} a_{n+1}}
$$

for all $n \in \mathbb{N}$. All of this can be found in Hardy and Wright [11].

We will also use some crude versions of Stirling's formula

$$
\begin{aligned}
n ! & =(n / e)^{n} e^{o(n)} \\
& =(n / e)^{n} e^{O(\log n)}
\end{aligned}
$$

and Abel's partial summation formula

$$
\sum_{j=1}^{n} x_{j} y_{j}=y_{n} \sum_{j=1}^{n} x_{j}-\sum_{k=1}^{n-1}\left(\left(y_{k+1}-y_{k}\right) \sum_{l=1}^{k} x_{l}\right) .
$$

\section{Results}

THEOREM 3.1. Let $Z_{1}<\cdots<Z_{K}$ be positive real numbers. Suppose that $\left\{A_{n}\right\}_{n=0}^{\infty}$ is a sequence consisting of numbers $Z_{1}, \ldots, Z_{K}$ such that every $Z_{j}, j=1, \ldots, K$, occurs infinitely many times. Let $\left\{a_{n}\right\}_{n=0}^{\infty}$ be a sequence of positive integers such that $a_{n}=A_{n}(1+$ $o(1))$ n. Set

$$
\begin{aligned}
& x=\liminf _{n \rightarrow \infty} \frac{\#\left\{j: A_{j}=Z_{K}, 1 \leq j \leq n\right\}}{n}, \\
& y=\limsup _{n \rightarrow \infty} \frac{\#\left\{j: A_{j}=Z_{1}, 1 \leq j \leq n\right\}}{n}, \\
& X=Z_{K}^{x} Z_{K-1}^{1-x}
\end{aligned}
$$

and

$$
Y=Z_{1}^{y} Z_{2}^{1-y}
$$

Let $\varepsilon$ be a positive real number. Then for all sufficiently large $N$ we have

$$
J_{a}(N)>\frac{(1-\varepsilon) \log (Y \log N /(e \log \log N))}{Z_{K} \log N}
$$

and for infinitely many $N$ we have

$$
J_{a}(N)<\frac{(1+\varepsilon) \log (X \log N /(e \log \log N))}{Z_{K} \log N} .
$$


Proof of Theorem 3.1. From the recurrence formulae of continued fractions (2) and the growth rate $a_{n}=A_{n}(1+o(1)) n$ we obtain

$$
q_{n}=a_{n} q_{n-1}+q_{n-2}=a_{n} q_{n-1}(1+o(1))=\cdots=e^{o(n)} \prod_{j=1}^{n} a_{j}=e^{o(n)} n ! \prod_{j=1}^{n} A_{j} .
$$

Now the proof of Theorem 3.1 falls into two cases.

First we prove upper bound (8). By the definition of $x$ we have the bounds

$$
Z_{1}^{n} \leq \prod_{j=1}^{n} A_{j} \leq Z_{K}^{n(x+o(1))} Z_{K-1}^{n(1-x+o(1))}=X^{n} e^{o(n)}
$$

Now applying Stirling's formula (4) to (9) gives

$$
\left(\frac{Z_{1}}{e}(1+o(1)) n\right)^{n} \leq q_{n} \leq\left(\frac{X}{e}(1+o(1)) n\right)^{n},
$$

noting that $(1+o(1))^{n}=e^{o(n)}$. This implies

$$
\begin{aligned}
n & \geq \frac{\log q_{n}}{\log \left(\frac{X}{e}(1+o(1)) n\right)} \geq \frac{\log q_{n}}{\log \left(\frac{X}{e}(1+o(1)) \frac{\log q_{n}}{\log \left(\frac{Z_{1}}{e}(1+o(1)) n\right)}\right)} \\
& \geq \frac{\log q_{n}}{\log \left(\frac{X}{e}(1+o(1)) \frac{\log q_{n}}{\log \left(\frac{Z_{1}}{e}(1+o(1)) \frac{\log q_{n}}{\log \left(\frac{X}{e}(1+o(1)) n\right.}\right)}\right)} \geq \frac{X \log q_{n}}{\log \left(\frac{\log \log q_{n}}{e}\right)+o(1)} .
\end{aligned}
$$

The last inequality is bounding $n=o\left(q_{n}\right)$ and simplifying. Consider only those values $N$ that equal some $q_{n}$ such that $A_{n+1}=Z_{K}$. By (3) we get

$$
J_{a}(N)<\frac{1}{a_{n+1}}=\frac{1}{A_{n+1}(n+1)(1+o(1))}=\frac{1+o(1)}{Z_{K} n},
$$

and so (8) follows from estimate (10).

Then we prove the lower bound (7). From (9) we obtain

$$
\left(\frac{Y}{e}(1+o(1)) n\right)^{n} \leq q_{n} \leq\left(\frac{Z_{K}}{e}(1+o(1)) n\right)^{n} .
$$

This yields

$$
\begin{aligned}
n & \leq \frac{\log q_{n}}{\log \left(\frac{Y}{e}(1+o(1)) n\right)} \leq \frac{\log q_{n}}{\log \left(\frac{Y}{e}(1+o(1)) \frac{\log q_{n}}{\log \left(\frac{Z_{K}}{e}(1+o(1)) n\right)}\right)} \\
& \leq \frac{\log q_{n}}{\log \left(\frac{Y}{e}(1+o(1)) \frac{\log q_{n}}{\log \left(\frac{Z_{K}}{e}(1+o(1)) \frac{\log q_{n}}{\log \left(\frac{Y}{e}(1+o(1)) n\right)}\right)}\right)} \leq \frac{Y \log q_{n}}{\log \left(\frac{\log q_{n}}{e \log q_{n}}\right)+o(1)} .
\end{aligned}
$$

As all of the best approximations of $a$ are the convergents (in fact, if $N$ is not a convergent then $J_{a}(N) \geq 1 / 2$ ), we may assume that $N$ equals $q_{n}$. As $A_{n+1} \leq Z_{K}$, we get

$$
J_{a}(N)>\frac{1}{a_{n+1}+2}=\frac{1}{A_{n+1}(n+1)(1+o(1))+2} \geq \frac{1+o(1)}{Z_{K} n},
$$

by (3) and so (7) follows from estimate (11). 
THEOREM 3.2. Let $Z_{1}<\cdots<Z_{K}$ be positive real numbers and $z_{1}, \ldots, z_{K}$ be nonnegative real numbers such that $\sum_{j=1}^{K} z_{j}=1$. Suppose that $\left\{A_{n}\right\}_{n=0}^{\infty}$ is a sequence consisting of the numbers $Z_{1}, \ldots, Z_{K}$ such that every $Z_{j}, j=1, \ldots, K$, occurs infinitely many times and has asymptotic density $z_{j}$. Let $\left\{a_{n}\right\}_{n=0}^{\infty}$ be a sequence of positive integers. Set

$$
Z=\prod_{j=1}^{K} Z_{j}^{z_{j}}
$$

If $a_{n}=A_{n}(1+o(1)) n$, then we have

$$
J_{a}(N)=\frac{(1+\bar{o}(1)) \log (Z \log N /(e \log \log N))}{Z_{K} \log N} .
$$

If there exists a positive real number $C$ such that $a_{n}=A_{n}\left(1+O\left(n^{-C}\right)\right) n$, then we have

$$
J_{a}(N)=\frac{\log (Z \log N /(e \log \log N))+\bar{o}(1)}{Z_{K} \log N} .
$$

Proof of Theorem 3.2. In both cases we have at least $a_{n}=A_{n}(1+o(1)) n$, so (9) is true. By Stirling's formula (4) and the fact that

$$
\prod_{j=1}^{n} A_{j}=Z_{1}^{n\left(z_{1}+o(1)\right)} \cdots Z_{K}^{n\left(z_{K}+o(1)\right)}=Z^{n} e^{o(n)}
$$

we obtain

$$
q_{n}=\left(\frac{Z}{e}(1+o(1)) n\right)^{n}
$$

Now

$$
n=\frac{\log q_{n}}{\log \left(Z \log q_{n} /\left(e \log \log q_{n}\right)\right)+o(1)}
$$

and in fact the whole first claim follows just as in the proof of Theorem 3.1.

Now suppose further that $a_{n}=A_{n}\left(1+O\left(n^{-C}\right)\right) n$ for some positive $C$. For the upper bound we again consider only those $N$ that equal some $q_{n}$ such that $A_{n+1}=Z_{K}$. Inequality (3) implies

$$
J_{a}(N)<\frac{1}{a_{n+1}}=\frac{1}{A_{n+1}\left(1+O\left(1 /(n+1)^{C}\right)\right)(n+1)}=\frac{1+O\left(1 / n^{\min \{C, 1\}}\right)}{Z_{K} n}
$$

so everything is fine after substitution of $n$. For the lower bound we make the justified assumption that $N$ equals some $q_{n}$, and the claim follows since (3) gives

$$
J_{a}(N)>\frac{1}{a_{n+1}+2}=\frac{1}{A_{n+1}\left(1+O\left(1 /(n+1)^{C}\right)\right)(n+1)+2} \geq \frac{1+O\left(1 / n^{\min \{C, 1\}}\right)}{Z_{K} n} .
$$

THEOREM 3.3. Let $Z_{1}<\cdots<Z_{K}$ be positive real numbers and $z_{1}, \ldots, z_{K}$ be nonnegative real numbers such that $\sum_{j=1}^{K} z_{j}=1$. Suppose that $\left\{A_{n}\right\}_{n=0}^{\infty}$ is a sequence consisting of the numbers $Z_{1}, \ldots, Z_{K}$ such that for every $Z_{j}, j=1, \ldots, K$ occurs infinitely many times and

$$
\#\left\{k: A_{k}=Z_{j}, 1 \leq k \leq n\right\}=z_{j} n+O\left(n^{1-C}\right)
$$


for some $C \in(0,1)$. Let $\left\{a_{n}\right\}_{n=0}^{\infty}$ be a sequence of positive integers such that $a_{n}=A_{n}(1+$ $\left.O\left(n^{-C}\right)\right) n$. Set

$$
Z=\prod_{j=1}^{K} Z_{j}^{z_{j}}
$$

Then

$$
J_{a}(N)=\frac{\log F_{k}(N)+\bar{O}(G(N))}{Z_{K} \log N},
$$

where $F_{k}(N)$ and $G_{k}(N)$ are defined recursively as $F_{0}(N)=\log N$,

$$
F_{k+1}(N)=\frac{Z \log N}{e \log F_{k}(N)}
$$

$G_{0}(N)=1$ and

$$
G_{k+1}(N)=\frac{G_{k}(N)}{\log F_{k}(N)} .
$$

Proof of Theorem 3.3. This time (9) will not be enough so we estimate

$$
\begin{aligned}
q_{n} & =a_{n} q_{n-1}+q_{n-2}=a_{n} q_{n-1}\left(1+O\left(\frac{1}{n^{2}}\right)\right)=\cdots=e^{O(1 / n)} \prod_{j=1}^{n} a_{j} \\
& =e^{O\left(n^{1-C}\right)} n ! \prod_{j=1}^{n} A_{j} .
\end{aligned}
$$

This is because by integrating we see that

$$
\log \prod_{j=1}^{n}\left(1+O\left(\frac{1}{j^{D}}\right)\right)=\sum_{j=1}^{n} \log \left(1+O\left(\frac{1}{j^{D}}\right)\right)=\sum_{j=1}^{n} O\left(\frac{1}{j^{D}}\right)=O\left(n^{1-D}\right)
$$

for any positive $D$ (here $D=2$ and $D=C$ ). Using Stirling's formula (5) and

$$
\prod_{j=1}^{n} A_{j}=Z_{1}^{z_{1} n+O\left(n^{1-C}\right)} \cdots Z_{K}^{z_{K} n+O\left(n^{1-C}\right)}=Z^{n} e^{O\left(n^{1-C}\right)}
$$

gives

$$
q_{n}=\left(\frac{Z}{e} n\right)^{n e^{O\left(n^{1-C}\right)}}
$$

This yields

$$
\begin{aligned}
& n=\frac{\log q_{n}}{\log \left(\frac{Z}{e} n\right)+O\left(\frac{1}{n^{C}}\right)}=\frac{\log q_{n}}{\log \left(\frac{Z \log q_{n}}{e \log \left(\frac{Z}{e} n\right)}\right)+O\left(\frac{1}{n^{C}}\right)} \\
& =\frac{\log q_{n}}{\log \left(\frac{Z \log q_{n}}{e \log \left(\frac{Z \log q_{n}}{e \log \left(\frac{Z}{e} n\right)}\right)}\right)+O\left(\frac{1}{n^{C}}\right)}=\cdots .
\end{aligned}
$$


After $k$ steps we make the estimate $n=O\left(\log q_{n}\right)$, to obtain

$$
n=\frac{\log q_{n}}{\log \left(F_{k}\left(q_{n}\right)\right)+O\left(G_{k}\left(q_{n}\right)\right)}
$$

via mathematical induction. Note that the new error $O\left(G\left(\log q_{n}\right)\right)$ is always bigger than the old $O\left(n^{-C}\right)$. The proof is finished in the usual manner using (3) and only considering those $N$ that equal some $q_{n}$ with $A_{n+1}=Z_{K}$ for the upper bound.

THEOREM 3.4. Let $\left\{V_{n}\right\}_{n=0}^{\infty}$ be a sequence of non-negative real numbers such that

$$
0<\liminf _{n \rightarrow \infty} \frac{1}{n} \sum_{j=1}^{n} V_{j} \leq \limsup _{n \rightarrow \infty} \frac{1}{n} \sum_{j=1}^{n} V_{j}<\infty .
$$

Let $\left\{a_{n}\right\}_{n=0}^{\infty}$ be a sequence of positive integers such that $a_{n}=n^{V_{n}}$.

If $\lim \sup _{n \rightarrow \infty} V_{n}=\alpha<\infty$, then

$$
J_{a}(N)=\left(\frac{\log \log N}{\log N}\right)^{\alpha+\bar{o}(1)} .
$$

If $\lim \sup _{n \rightarrow \infty} V_{n} / \log n=\beta<\infty$, then

$$
J_{a}(N)=\left(\frac{\log \log N}{\log N}\right)^{(\beta+\bar{o}(1)) \log (\log N / \log \log N)} .
$$

Proof of Theorem 3.4. By Abel's partial summation formula (6)

$$
\begin{aligned}
\sum_{j=1}^{n} V_{j} \log j & =\log n \sum_{j=1}^{n} V_{j}-\sum_{k=1}^{n-1}\left((\log (k+1)-\log k) \sum_{l=1}^{k} V_{l}\right) \\
& =\log n \sum_{j=1}^{n} V_{j}+O(n) .
\end{aligned}
$$

From this, the recurrence formulae of continued fractions (2) and the growth rate $a_{n}=n^{V_{n}}$, we obtain that

$$
q_{n}=a_{n} q_{n-1}+q_{n-2}=a_{n} q_{n-1} O(1)=\cdots=e^{O(n)} \prod_{j=1}^{n} a_{j}=n^{n \varepsilon_{1}(n)},
$$

where $0<a_{1} \leq \varepsilon_{1}(n) \leq b_{1}<\infty$. This yields

$$
n=\frac{\log q_{n}}{\varepsilon_{1}(n) \log n}=\frac{\log q_{n}}{\varepsilon_{1}(n) \log \left(\log q_{n} /\left(\varepsilon_{1}(n) \log n\right)\right)}=\frac{\log q_{n}}{\varepsilon_{2}(n) \log \log q_{n}},
$$

where $0<a_{2} \leq \varepsilon_{2}(n) \leq b_{2}<\infty$. This and (3) imply (12) and (13), if in the upper bounds we only consider those $N$ equal to some $q_{n}$ such that $V_{n+1}$ is sufficiently close to $\alpha$ and $\beta \log n$, respectively.

THEOREM 3.5. Let $\beta$ and $\gamma$ be positive real numbers. Let $\left\{V_{n}\right\}_{n=0}^{\infty}$ be a sequence of nonnegative real numbers such that

$$
\lim _{n \rightarrow \infty} \frac{1}{n} \sum_{j=1}^{n} V_{j}=\gamma
$$


and for infinitely many $k$ we have $V_{k}=\beta=\max _{n \in \mathbb{N}} V_{n}$. Suppose that $\left\{A_{n}\right\}_{n=0}^{\infty}$ is a sequence of positive real numbers such that

$$
0<\alpha_{1} \liminf _{n \rightarrow \infty} A_{n} \leq \limsup _{n \rightarrow \infty} A_{n}=\alpha_{2}<\infty .
$$

Let $\left\{a_{n}\right\}_{n=0}^{\infty}$ be a sequence of positive integers such that $a_{n}=A_{n} n^{V_{n}}$. Let $\varepsilon$ be a positive real number. Then for all sufficiently large $N$ we have

$$
J_{a}(N)>\frac{(1-\varepsilon)(\gamma \log \log N / \log N)^{\beta}}{\alpha_{2}}
$$

and for infinitely many $N$ we have

$$
J_{a}(N)<\frac{(1+\varepsilon)(\gamma \log \log N / \log N)^{\beta}}{\alpha_{1}} .
$$

Proof of Theorem 3.5. We use Abel's summation formula (6) as in the proof of Theorem 3.5, taking into account that now

$$
\sum_{j=1}^{n} V_{j}=\gamma n+o(n)
$$

to see

$$
q_{n}=n^{\gamma n+o(n)}
$$

and

$$
n=\frac{\log q_{n}}{\gamma(1+o(1)) \log \log q_{n}} .
$$

This and (3) imply (14) and (15) if we only consider those $N$ equal to some $q_{n}$ such that $V_{n+1}=\beta$ for the upper bound.

Proof of Theorem 1.1. Theorem 1.1 is a consequence of Theorem 3.5.

THEOREM 3.6. Set $a=\left[1 ; \overline{2}^{d(\lambda)}\right]_{\lambda=1}^{\infty}$. Then

$$
J_{a}(N)=2^{-2^{\log \log N /(\log \log \log N-1+\bar{\sigma}(1))}} .
$$

Proof of Theorem 3.6. From the fact that

$$
\sum_{j=1}^{n} d(j)=n \log n+O(n)
$$

(see Hardy and Wright [11, p. 347]) and the recurrence formulae (2) we get

$$
q_{n}=a_{n} q_{n-1}+q_{n-2}=a_{n} q_{n-1} O(1)=\cdots=e^{O(n)} \prod_{j=1}^{n} a_{j}=e^{\log (2) n \log n+O(n)} .
$$

This yields

$$
\begin{aligned}
n & =\frac{\log q_{n}}{\log (2) \log n+O(1)}=\frac{\log q_{n}}{\log (2) \log \left(\log q_{n} / \log (2) \log n+O(1)\right)+O(1)} \\
& =\frac{\log q_{n}}{\log (2) \log \log q_{n}-\log (2) \log \log n+O(1)} \\
& =\frac{\log q_{n}}{\log (2) \log \log q_{n}-\log (2) \log \log \log q_{n}+O(1)} .
\end{aligned}
$$


From this, (3) and the fact that for every positive real number $\varepsilon$ we have

$$
d(n) \leq 2^{\log n /(\log \log n-1-\varepsilon)}
$$

(see Nicolas [15]) we obtain the lower bound.

For the upper bound consider only those $N$ that equal some $q_{n}$, where $n+1$ is the product of $k$ smallest prime numbers $p_{j}$. Now $d(n+1)=2^{k+1}$, so we need to express $k$ in terms of $n$. By using the prime number theorem $p_{n} /(n \log n)=1+o(1)$ we see that

$$
\log (n+1)=\sum_{j=1}^{k} \log p_{j}=\sum_{j=1}^{k} \log j+\sum_{j=1}^{k} \log \log j+o(k) .
$$

Stirling's formula (4) gives

$$
\sum_{j=1}^{k} \log j=k \log k-k+o(\log k)=k \log k-k+o(k)
$$

and Abel's partial summation formula (6) gives

$$
\sum_{j=1}^{k} \log \log j=k \log \log k+O(\operatorname{li}(k))=k \log \log k+o(k),
$$

and after solving for $k$ and simplifying we obtain

$$
k=\frac{\log n}{\log \log n-1+o(1)} .
$$

The claim now follows from (3) and (16).

THEOREM 3.7. Let $a_{0}$ be an integer and $a_{1}, \ldots, a_{s}, b$ be positive integers such that $b>1$. Set $a=[a_{0} ; a_{1}, \ldots, a_{s}, \underbrace{\overline{b^{\lambda}, \ldots, b^{\lambda}}}_{m \text { times }}]_{\lambda=1}^{\infty}$. Then

$$
J_{a}(N)=\frac{1+O\left(b^{-2 \sqrt{2 \log N /(m \log b)}}\right)}{b^{1 / 2+\sqrt{1 / 4+2(\log N-C) /(m \log b)}}},
$$

where

$$
e^{C}=\left(\prod_{j=1}^{s} a_{j}\right)\left(\prod_{j=2}^{\infty}\left(1+\frac{q_{n-2}}{a_{n} q_{n-1}}\right)\right) .
$$

Proof of Theorem 3.7. Since $a_{n+1}=b^{[(n-s) / m]+1}$, it is enough by (3) to only look at $n$ such that $n \equiv s(\bmod m)$. Now recurrence formulae (2) yield

$$
\begin{aligned}
q_{n} & =a_{n} q_{n-1}+q_{n-2}=a_{n} q_{n-1}\left(1+\frac{q_{n-2}}{a_{n} q_{n-1}}\right)=\cdots=a_{1} \prod_{j=2}^{n} a_{j}\left(1+\frac{q_{j-2}}{a_{j} q_{j-1}}\right) \\
& =e^{C}\left(\prod_{j=n+1}^{\infty}\left(1+\frac{q_{j-2}}{a_{j} q_{j-1}}\right)\right)^{-1} \prod_{j=s+1}^{n} a_{j}=e^{C+O\left(b^{-2 n / m}\right)} \prod_{j=s+1}^{n} a_{j} .
\end{aligned}
$$


Hence

$$
\begin{aligned}
\log q_{n} & =C+O\left(b^{-2 n / m}\right)+m \log b \sum_{j=1}^{(n-s) / m} j \\
& =C+O\left(b^{-2 n / m}\right)+\log b \frac{(n-s)(n-s+m)}{2 m} .
\end{aligned}
$$

Therefore,

$$
\begin{aligned}
n & =s-\frac{m}{2}+\sqrt{\frac{m^{2}}{4}+\frac{2 m}{\log b}\left(\log q_{n}-C+O\left(b^{-2 n / m}\right)\right)} \\
& =s-\frac{m}{2}+\sqrt{\frac{m^{2}}{4}+\frac{2 m}{\log b}\left(\log q_{n}-C+O\left(b^{-2 \sqrt{2 \log q_{n} /(m \log b)}}\right)\right.} .
\end{aligned}
$$

This and the fact that $a_{n+1}=b^{(n-s) / m+1}$ imply that for $n \equiv s(\bmod m)$ we have

$$
\begin{aligned}
J_{a}(N) & =\frac{1+O\left(b^{-2 n / m}\right)}{a_{n+1}}=\frac{1+O\left(b^{-2 \sqrt{2 \log q_{n} /(m \log b)}}\right)}{b^{[(n-s) / m]+1}} \\
& =\frac{1+O\left(b^{-2 \sqrt{2 \log q_{n} /(m \log b)}}\right)}{b^{1 / 2+\sqrt{1 / 4+2\left(\log q_{n}-C\right) /(m \log b)}}}
\end{aligned}
$$

and (17) follows.

Acknowledgements. This work was supported by the European Regional Development Fund in the IT4Innovations Centre of Excellence project (CZ.1.05/1.1.00/02.0070) and by the grant no. P201/12/2351. Kalle Leppälä was supported by Academy of Finland, grant 138522.

\section{REFERENCES}

[1] J. M. Borwein, P. B. Borwein. Pi and the AGM. A Study in Analytic Number Theory and Computational Complexity (Canadian Mathematical Society Series of Monographs and Advance Texts). Wiley, New York, 1987.

[2] P. Bundschuh. Irrationalitätsmasse für $e^{a}, a \neq 0$ rational oder Liouville-Zahl, Math. Ann. 192 (1971), 229242.

[3] P. Bundschuh. On simple continued fractions with partial quotients in arithmetic progressions. Lithuanian Math. J. 38(1) (1998), 15-26.

[4] L. Euler. De fractionibus continuis dissertatio. Comm. Acad. Sci. Petropolitanae 9 (1737), 98-137; Opera Omnia, Ser. 1, XIV, 186-215.

[5] N. I. Fel'dman and Yu. V. Nesterenko. Transcendental Numbers, Number Theory IV (Encyclopaedia of Mathematical Sciences, 44). Springer, Berlin, 1998, pp. 1-345.

[6] A. I. Galochkin. The sharpening of the bounds for certain linear forms. Math. Notes 20(1) (1976), 35-45.

[7] J. Hančl, M. Leinonen, K. Leppälä and T. Matala-aho. Explicit irrationality measures for continued fractions. J. Number Theory 132(8) (2012), 1758-1769.

[8] J. Hančl and K. Leppälä. Irrationality measures for continued fractions with arithmetic functions. Publ. l'Inst. Math. (Beograd) 97(111) (2015), 139-148.

[9] J. Hančl, S. Dodulíková, O. Kolouch, M. Leinonen and K. Leppälä. Irrationality measures for almost periodic continued fractions. Georgian Math. J. 23(1) (2016), 55-67, doi:10.1515/gmj-2015-0056.

[10] J. Hančl, J. Šustek and A. Jaššová. Lebesgue measure and Hausdorff dimension of special sets of real numbers from (0, 1). Ramanujan J. 28(1) (2012), 15-23. 
[11] G. H. Hardy and E. M. Wright. An Introduction to the Theory of Numbers, 6th edn. Oxford University Press, Oxford, 2008. Revised by D. R. Heath-Brown and J. H. Silverman, with a foreword by Andrew Wiles.

[12] M. Hata. A lower estimate for $\left\|e^{n}\right\|$. J. Number Theory 130(8) (2010), 1685-1704.

[13] T. Komatsu. A Diophantine approximation of $e^{1 / s}$ in terms of integrals. Tokyo J. Math. 32(1) (2009), 159176.

[14] K. Mahler. Zur Approximation der Exponentialfunktion und des Logarithmus, Teil I. J. Reine Angew. Math. 166 (1932), 118-136.

[15] J. L. Nicolas. Bornes effectives pour certaines fonctions arithmétiques (in French) [Effective bounds for certain arithmetic functions]. Colloque de Théorie Analitique des Nombres "Jean Coquet" (Marseille, 1985) (Publications Mathématiques d'Orsay, 88-02). Université Paris XI, Orsay, 1988, 91-95.

[16] O. Perron. Über eine spezielle Klasse von Kettenbrüchen. Rend. Pal. 29 (1910).

[17] I. Shiokawa. Rational approximations to the values of certain hypergeometric functions. Number Theory and Combinatorics, Japan, 1984 (Tokyo, Okayama and Kyoto, 1984). World Scientific, Singapore, 1985, pp. 353-367.

[18] J. Sondow. A geometric proof that $e$ is irrational and a new measure of its irrationality. Amer. Math. Monthly 113(7) (2006), 637-641.

[19] B. G. Tasoev. Rational approximations to certain numbers. Math. Notes 67(6) (2000), 786-791.

\author{
J. Hančl \\ Department of Mathematics, Faculty of Science and Centre of Excellence IT4Innovation \\ Division of $U O$ \\ Institute for Research and Applications of Fuzzy Modeling \\ University of Ostrava \\ 30. dubna 22, 70103 Ostrava 1, Czech Republic \\ (E-mail:hancl@osu.cz)
}

\author{
K. Leppälä \\ Bioinformatics Research Centre \\ Aarhus University \\ 8000 Aarhus C, Denmark \\ (E-mail: kalle.m.leppala@gmail.com)
}

